\title{
PRESSURE DROP IN A SPOUTED BED OF CORK STOPPERS
}

\author{
A. Magalhães ${ }^{\mathrm{a}}$ \\ and C. Pinhob \\ ${ }^{\mathrm{a}}$ INEGI \\ Rua do Barroco, 174 \\ 4465-591 Leça do Balio, Portugal \\ nicola@tvtel.pt \\ ${ }^{\mathrm{b}}$ CEFT-FEUP \\ Universidade do Porto \\ Rua Dr. Roberto Frias s/n \\ 4200-465 Porto, Portugal \\ ctp@fe.up.pt \\ Corresponding author \\ ABSTRACT \\ The spouted bed is a particular type of fluidized bed which is well suited for \\ the handling of large size particles like cork stoppers. It is commonly used \\ for the drying of granulates and the present work is the first part of a study \\ concerning the drying of cork stoppers in a laboratory scale spouted bed \\ reactor. The study considers two stoppers size, one the port wine stopper \\ and another small size stopper. The choice of these smaller sizes was \\ imposed by the small dimensions of the reactor. In this preliminary set of \\ experiments the fluid-dynamics regime of the particular two phase flow was \\ characterized. For different experimental situations covering several air \\ flows and temperatures typical of cork drying operations the evolution of \\ pressure drop was evaluated and a correlation for the minimum spouting \\ velocity was obtained. \\ Keywords: spouted bed , minimum spouting velocity, pressure drop , cork \\ stoppers.
}

\section{NOMENCLATURE}

Ar Archimedes number, Eq. (5)

$d_{p} \quad$ average deviation, Eq. (6)

$d_{p} \quad$ particle diameter, $\mathrm{m}$

$d_{s p h} \quad$ diameter of a sphere with the same volume as the stopper, $\mathrm{m}$

$D$ bed diameter, $\mathrm{m}$

$D_{i} \quad$ diameter of jet entrance, $\mathrm{m}$

$f \quad$ generic function, $\mathrm{Eq}(2)$

$g \quad$ gravitational constant, $\mathrm{m} \mathrm{s}^{-2}$

$H$ bed height, $\mathrm{m}$

$L \quad$ stopper length, $\mathrm{m}$

$N \quad$ number of experiments

$p$ pressure, $\mathrm{Pa}$

$R e_{\text {cal }}$ Calculated Reynolds number, Eq. (6)

$R e_{\text {exp }}$ Experimental Reynolds number, Eq. (6)

$R e_{m s}$ Reynolds number for minimum spouting conditions, Eq. (4)

$U_{m s} \quad$ minimum spouting velocity, $\mathrm{m} \mathrm{s}^{-1}$

$\varnothing \quad$ stopper diameter, $\mathrm{m}$

\section{Greek symbols}

$\rho_{f} \quad$ fluid density, $\mathrm{kg} / \mathrm{m}^{3}$

$\rho_{p} \quad$ particle density, $\mathrm{kg} / \mathrm{m}^{3}$

$\mu_{\mathrm{f}} \quad$ fluid viscosity, $\mathrm{N} \mathrm{s} \mathrm{m}^{-2}$

$\phi \quad$ sphericity of the stopper

\section{INTRODUCTION}

The non conventional type of fluidized bed known as spouted bed (Mathur and Esptein, 1974) is a technique commonly used for drying of solids
(Németh et al., 1983; Viswanathan, 1986) or for handling of solids in another technological processes (Mathur and Epstein, 1974; Markowski and Kaminski, 1983; Olazar et al., 1993; Yang, 2003). The drying of cork stoppers is an important step in their production cycle involving usually fixed bed drying processes with the all encompassing questions concerning mass transfer (Martins et al., 1994; Pinho and Santos, 1997) and pressure drop problems (Rangel et al., 2001, 2001a). The spouted bed drying of cork stoppers is a particular situation only used for the processing of very small batches of stoppers to fulfill some special orders of high grade costumers. The approach that has been followed so far by equipment builders was purely empirical and the absence of a minimum of reliable scientific and technical data lead to the development of this small research work on the characterization of jet spouted bed drying of cork stoppers. Previously to the drying experiments, it was necessary to characterize the fluidization characteristics of this type of bed. This paper resumes some of the conclusions obtained for the study of the minimum spouting velocity of a laboratory scale jet spouted fluidized bed, with small size cork stoppers.

The measurement of the minimum spouting velocity has been the subject of many studies and consequently experimental data correlations were developed. In the book of Mathur and Epstein (1974) several correlations were presented and discussed. However, some researchers found that the available correlations could not be applied in some particular situations. The jet spouted bed is one of such cases, where a primary correlation was proposed by Markowski and Kaminski (1983). These authors 
recommend that shallower spouted beds, with an important conical section, may increase solids circulation rate and thus augment the drying rate of the particles. The fact that a conical sector may have a great importance on the hydrodynamic behavior of the bed is then an aspect to take into account (Kmieg, 1983). Although some works pretend to obtain a general correlation for the minimum spouting velocity (Lefroy and Davidson, 1969; Littman and Morgan III, 1986), it is felt that the appropriate correlation for the minimum spouting velocity will always be highly dependent upon the combination of the bed geometry and the dimensional and physical properties of the particles being handled. Nothing was found in the literature for the fluidization of cork stoppers and then the general recommendations of Markowski and Kaminski (1983) were followed for the definition of the experimental set up concerning these drying studies

\section{EXPERIMENTAL DETAILS}

The experiments were carried out in a laboratory spouted bed installation composed by a centrifugal fan to supply the fluidizing air, a calibrated orifice plate for the flow measurement, an electrical heater composed by three $3 \mathrm{~kW}$ electrical resistances and the bed itself. Figure 1 presents an overall view of the experimental set up while in Fig. 2 are shown the main dimensions of the bed.

As referred in the introduction, to define the experimental setup the recommendations of Markowski and Kaminski (1983) were adopted and although it can be seen from Fig. 2 that the size of the conical part of the bed is small compared with the cylindrical region, a shallow bed of stoppers was always used. Maximum bed height above the conical part was only $180 \mathrm{~mm}$. Cork stoppers are rather large particles but with low density and consequently as soon as they are fluidized they are projected by the jet flow high above the bed, in a such a way that the installation of a secondary cylindrical part of $500 \mathrm{~mm}$ length, above the original cylindrical portion, see Figs. 1 and 2, was required with the sole purpose of keeping the stoppers inside the reactor. The experimental layout can be observed in Fig. 1. A bag made of stainless steel mesh contained the particles and allowed their simple extraction from the bed, later during the drying experiments. However, to be coherent with these later experiments, the characterization of spouting conditions was also carried out with such bag, Fig. 3.

A data acquisition system installed in a personal computer was connected to differential pressure transducers measuring the pressure drop in the orifice plate for flow rate calculations, as well as to differential pressure transducers installed in the spouted bed, to measure the evolution of pressure differentials as the fluidization was carried out. Fluid temperatures were measured through $\mathrm{T}$ type thermocouples. Temperatures were measured for the ambient air, air flow between the centrifugal fan and the orifice plate, hot air between the electrical heater and the spouted bed and in the spouted bed. The influences of ambient temperature and pressure fluctuations upon the measured value of the fluidizing air flow rate were accounted for through calibration procedures.

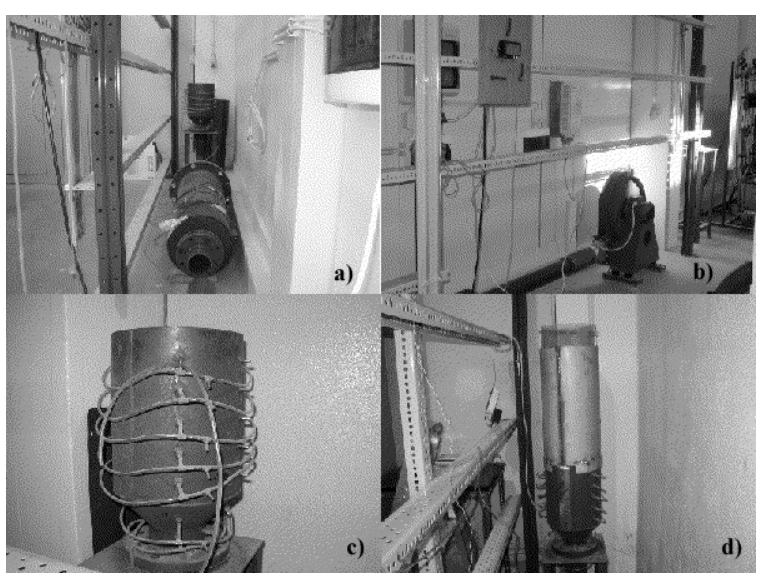

Figure 1. Images of the experimental lay-out: a) Air preheater and spouted bed; b) Centrifugal fan, frequency variator and diaphragm flow meter; c)

Close-up of the spouted bed and d) Cylindrical protection placed above the bed.

The data acquisition system had an internal board connected to two external boards for signal conditioning. All the boards were from Advantech. The used acquisition software was the GENIE. release 2.12, also from Advantech. The internal board reference PCL-818L had eight differential analog inputs and a resolution of 12 bits. The differential pressure transducers were connected to a PCLD-8115 external board and the thermocouples to a PCLD-789D external board, equipped with cold junction temperature compensation.

Pressure measurements were carried out with differential pressure transducers from Series $\mathrm{T}$ of Modus Instruments, Inc. Two different transducers types were used, according to the pressure range being measured. Transducers T1008EXB were for -1 $\mathrm{kPa}$ to $+1 \mathrm{kPa}$ pressure range, while transducers T1009EXB were for $-2 \mathrm{kPa}$ to $+2 \mathrm{kPa}$ pressure range. These transducers have an accuracy of $\pm 1 \%$ of span including linearity and hysteresis. Their output signal of $0-10 \mathrm{VDC}$ was send to the data acquisition board. The transducers outputs were checked towards U-type water manometer readings.

To change the air flow rate sent to the spouted bed, the centrifugal fan was controlled by means of a frequency variator from $\mathrm{ABB}$, model ACS401000932, with a resolution of $0.1 / 50 \mathrm{~Hz}$. 


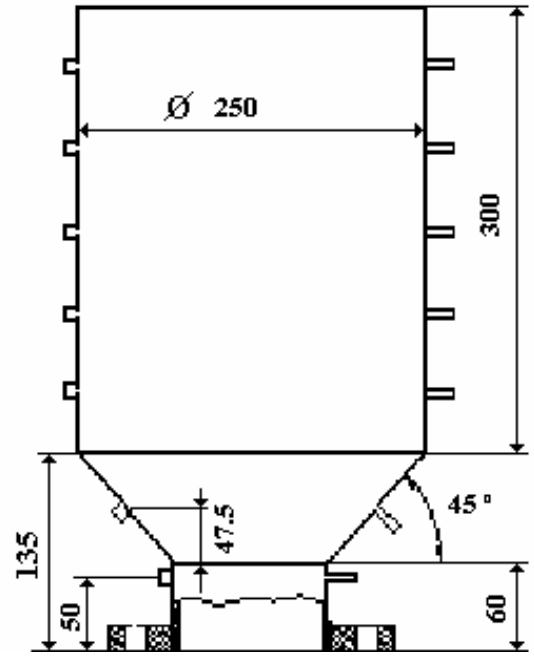

Figure 2. General dimensions (in $\mathrm{mm}$ ) of the spouted bed reactor.



Figure 3. The spouted bed of cork stoppers viewed from above.

The spouted bed was equipped with tapings in the cylindrical entrance pipe, on the conical and on the main cylindrical body to measure static pressures. For the determination of minimum spouting conditions pressures were measured on the entrance cylindrical pipe, which had an internal diameter of $114.8 \mathrm{~mm}, 50 \mathrm{~mm}$ above the flange, Fig. 2. The diameter of the jet was defined by an orifice plate placed above the flange, and two diameters, 50 and $60 \mathrm{~mm}$, were tested.

Alternative bed pressure measurements were also carried out using the pressure tapping placed on the conical part of the bed, but in the tested situations a strong recirculation region was formed and pressure readings were erroneous.

The maximum global uncertainty for the pressure drop measurements was of $4.4 \%$ whereas for temperature measurements it was of $3.3 \%$. These values include the complete measurement chain from the transducer to the final output in the computer monitor.

\section{EXPERIMENTAL RESULTS}

The measurements were carried out at atmospheric pressure and as referred in Tab. (1) two stopper sizes were used. The $29 \times 21(\mathrm{~mm} \times \mathrm{mm}-$ length $\mathrm{x}$ diameter) are Port wine stoppers. They are smaller than standard stoppers, and to be used on their proper function, each stopper is equipped with a plastic cap, although in these experiments such cap was absent. The other size $39 \times 22(\mathrm{~mm} \times \mathrm{mm})$ is one of the smallest standard sizes used by the industry. The choice of the smaller stopper sizes was imposed by the use of a smaller and cheaper experimental rig. The temperature of fluidizing air was in the range of 20 to $60{ }^{\circ} \mathrm{C}$. For the drying of cork stoppers temperatures above $80{ }^{\circ} \mathrm{C}$ are excluded to avoid the damage of the cork, thus the working temperature range was chosen to respect such limitations.

Table 1. Characteristics of tested stoppers.

\begin{tabular}{|c|c|c|}
\hline $\begin{array}{c}\text { Cork stopper } \\
\begin{array}{c}L \times \varnothing \\
(\mathrm{mm} \times \mathrm{mm})\end{array}\end{array}$ & $29 \times 21$ & $38 \times 22$ \\
\hline $\begin{array}{c}\text { Equivalent } \\
\text { diameter } \\
(\mathrm{mm})\end{array}$ & 26.8 & 30.2 \\
\hline $\begin{array}{c}\text { Sphericity } \\
(-)\end{array}$ & 0.866 & 0.846 \\
\hline $\begin{array}{c}\text { Density } \\
\left(\mathrm{kg} / \mathrm{m}^{3}\right)\end{array}$ & 164.8 & 177.7 \\
\hline $\begin{array}{c}\text { Particle diameter } \\
(\mathrm{mm})\end{array}$ & 23.2 & 25.5 \\
\hline
\end{tabular}

The dimension of the particles are fundamental to guarantee the obtainment of a spouted bed because the particles must belong to the group $\mathrm{D}$ of the Geldart classification (Geldart, 1986). The border between group $\mathrm{B}$ and $\mathrm{D}$ is given by,

$$
\left(\rho_{p}-\rho_{f}\right) d_{p}{ }^{1.24}>0.23
$$

and applying this expression to the stoppers tested at ambient conditions $\left(1 \mathrm{~atm}\right.$ and $20{ }^{\circ} \mathrm{C}$ ) it is found that for the cork wine stoppers $29 \times 21(\mathrm{~mm} \times \mathrm{mm})$ that the left hand side of the previous inequality gives 1.84 whereas for the larger stoppers $(39 \times 22-\mathrm{mm} \times$ $\mathrm{mm}$ ), the result is of 2.3 , meaning that in both tested sizes the particles belonged to the group D of the above mentioned Geldart classification

Two inlet jet diameters, 50 and $60 \mathrm{~mm}$, and four bed heights of $100,150,200$ and $250 \mathrm{~mm}$ were tested. As the height of the conical part of the bed available for the particles is of $70 \mathrm{~mm}$, due to a $5 \mathrm{~mm}$ gap imposed by the stainless steel mesh, instead of the value that could be obtained from Fig. 2 which would be of $135-60=75 \mathrm{~mm}$, if such gap was not accounted for, the corresponding heights in the circular region of the bed were respectively of 30,80 , 130 and $180 \mathrm{~mm}$. Consequently it could be assumed that the tested beds were shallow beds. 
Due to the low density of the particles and the reduced bed height, the evolution of the pressure drop is different from what is usually found in classical and jet spouted beds of granular materials. In the present situation it was not observed the sudden jump in the pressure drop curve corresponding to the transition from fixed bed towards spouted bed conditions. The transition condition is detected through a slight change on the slope of the pressure drop curve and a simultaneous close visual inspection of the bed behavior is recommended.

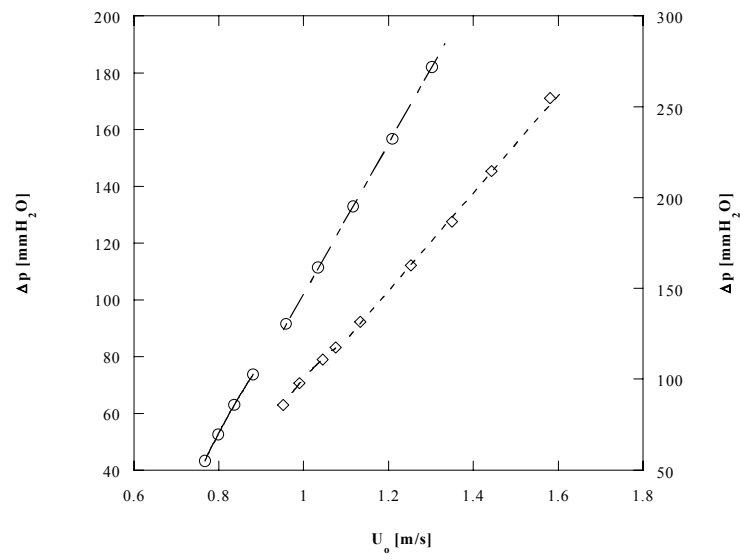

Figure 4. Pressured drop versus superficial gas velocity for the spouted bed of $29 \times 21$ cork stoppers. Jet diameter of $50 \mathrm{~mm}$. $\bigcirc 40{ }^{\circ} \mathrm{C}$ and $H=150 \mathrm{~mm}$, left axis; $\diamond 60^{\circ} \mathrm{C}$ and $H=200 \mathrm{~mm}$, right axis.

Figures 4 and 5 show typical pressure drop versus gas superficial velocity curves for the jet spouted bed of cork stoppers of $29 \times 21 \mathrm{~mm} \times \mathrm{mm}$ size, for two different jet diameters, 50 and $60 \mathrm{~mm}$.

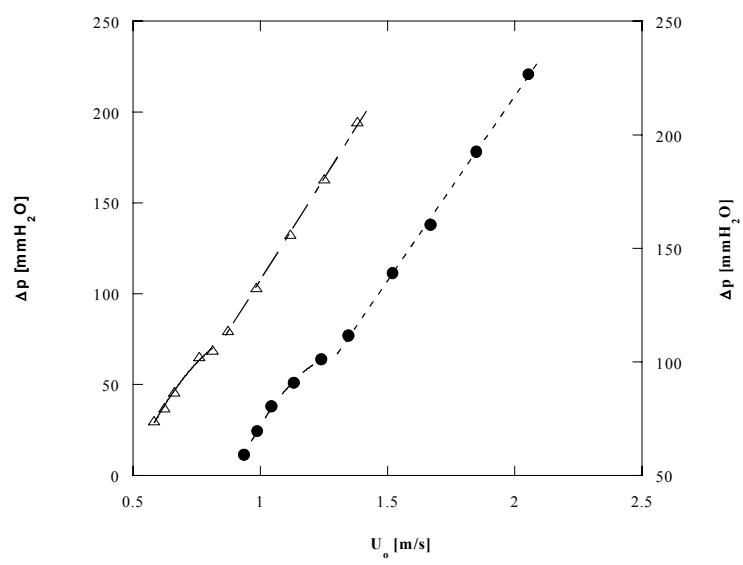

Figure 5. Pressured drop versus superficial gas velocity for the spouted bed of $29 \times 21$ cork stoppers. Jet diameter of $60 \mathrm{~mm} . \triangle 30^{\circ} \mathrm{C}, H=200 \mathrm{~mm}$, left axis; $60{ }^{\circ} \mathrm{C}, H=250 \mathrm{~mm}$, right axis.

In Fig. 4 for both curves, the first four points are for the packed bed, whereas the following points are for the spouted bed. The inflexion point is between these two regions and can be obtained through the extrapolation of the two trend lines that are shown for each situation. The interception of the trend lines defines graphically the minimum spouting velocity. Such interception is best seen from Fig. 5 Here, the curve for $30{ }^{\circ} \mathrm{C}$ has four points before fluidization while the curve for $60{ }^{\circ} \mathrm{C}$ has five points before fluidization. However, in both situations shown in Fig. 5 the slope difference is more detectable. Trend lines before fluidization are quadratic curves, while after fluidization are linear.

Pressure versus gas flow rate data for each experimental run were analyzed in a work sheet and the corresponding minimum spouting velocities were determined as mentioned.

\section{CORRELATION FOR MINIMUM SPOUTING VELOCITY}

Through dimensionless analysis it is concluded that by taking into account the main physical parameters characterizing the phenomenon, a generic relation defining the minimum spouting velocity should be like

$$
\frac{U_{m s} d_{p} \rho_{f}}{\mu_{f}}=f\left[\left(\frac{d_{p}}{D}\right),\left(\frac{D_{i}}{D}\right),\left(\frac{H}{D}\right),\left(\frac{\rho_{p}}{\rho_{f}}\right),\left(\frac{g d_{p}^{3} \rho_{f}\left(\rho_{p}-\rho_{f}\right)}{\mu_{f}{ }^{2}}\right)\right]
$$

where $U_{m s}$ is the minimum spouting velocity (superficial velocity) calculated for the cylindrical portion of the bed, $d_{p}=\phi d_{s p h}$ is the particle diameter, $D_{i}$ is the internal diameter of the jet, $D$ is the internal diameter of the cylindrical portion of the bed, $H$ is the bed height, $\rho_{p}$ is the particle density, $g$ is the gravitational constant, $\rho_{f}$ and $\mu_{f}$ are respectively the air density and dynamic viscosity. $\phi$ is the sphericity of the stopper and $d_{s p h}$ is the diameter of the sphere with the same volume as the stopper. As in the experiments only cork particles, i.e. stoppers, were used, the ratio $\left(\rho_{p} / \rho_{f}\right)$ was not considered relevant for the determination of the correlation and was discarded.

This approach has been followed by many authors (Mathur and Epstein, 1974; Markowski and Kaminski, 1983; Costa and Taranto, 2003) and a simple function obtained after numerical treatment of the present experimental data is.

$$
\operatorname{Re}_{m s}=0.3436\left(\frac{d_{p}}{D}\right)^{0.1006}\left(\frac{D_{i}}{D}\right)^{0.7873}\left(\frac{H}{D}\right)^{0.4812} A r^{0.5554}
$$

In this equation,

$$
R e_{m s}=\frac{U_{m s} d_{p} \rho_{f}}{\mu_{f}}
$$


is the Reynolds number for minimum spouting conditions while,

$$
A r=\frac{g d_{p}^{3} \rho_{f}\left(\rho_{p}-\rho_{f}\right)}{\mu_{f}^{2}}
$$

is the Archimedes number. In Eq. (2) the multiplying factor as well as the exponents of the dimensionless numbers are fitting parameters obtained through the application of a commercial software for nonlinear regression analysis NLREG. These parameters guarantee the best fit to the set of provided experimental data obtained in the experiments.

The comparison between experimental values of $\mathrm{Re}_{m s}$ and the corresponding values obtained through Eq. (2) is shown in Fig. 6. On this figure the straight line represents the correlation while the white squares are the corresponding experimental results. The closeness between experimental and calculated points is evident, the larger relative error obtained is of $11.5 \%$ while the average deviation calculated through

$$
d_{\text {avg }}=\sqrt{\frac{\sum\left(\frac{R e_{c a l}-R e_{\text {exp }}}{R e_{\text {exp }}}\right)^{2}}{N}}
$$

as suggested by Wen and Chen (1982), was of $5.2 \%$.

The range of application of the correlation is referred in Tab. 2. The temperature range for the fluidizing air went from 20 to $60{ }^{\circ} \mathrm{C}$ and all the experiments were carried out at atmospheric pressure.

Table 2. Range of dimensionless numbers.

\begin{tabular}{|l|c|c|}
\hline $\mathrm{Re}_{m s}$ & $1.00 \times 10^{3}$ & $3.12 \times 10^{3}$ \\
\hline$\left(d_{p} / D\right)$ & $9.28 \times 10^{-2}$ & $1.02 \times 10^{-1}$ \\
\hline$\left(D_{i} / D\right)$ & 0.20 & 0.24 \\
\hline$(H / D)$ & 0.40 & 1.00 \\
\hline $\mathrm{Ar}$ & $4.86 \times 10^{7}$ & $9.99 \times 10^{7}$ \\
\hline
\end{tabular}

The temperature of the fluidizing air affects its density and dynamic viscosity and consequently the $\operatorname{Re}_{m s}$ and $\mathrm{Ar}$ were calculated with the effective value of this temperature.

Owing to the shallow depth of the bed and the fact that stoppers are thrown above the bed as fluidization starts, the definition of a clear bed upper surface was impossible. Thus the definition of a critical or minimum spouting bed voidage was not viable and as such, this parameter was not taken into account in the correlation for the minimum spouting velocity, as commonly found in many experimental correlations (Mathur and Epstein, 1974; Markowski and Kaminski, 1983; Olazar et al., 1993; Costa and Taranto, 2003; Yang, 2003). However, for the corresponding fixed bed conditions, typical values for bed voidage were of 0.386 for the $29 \times 21$ $(\mathrm{mm} \times \mathrm{mm})$ stoppers and of 0.544 for the $38 \times 22$ $(\mathrm{mm} \times \mathrm{mm})$ stoppers.

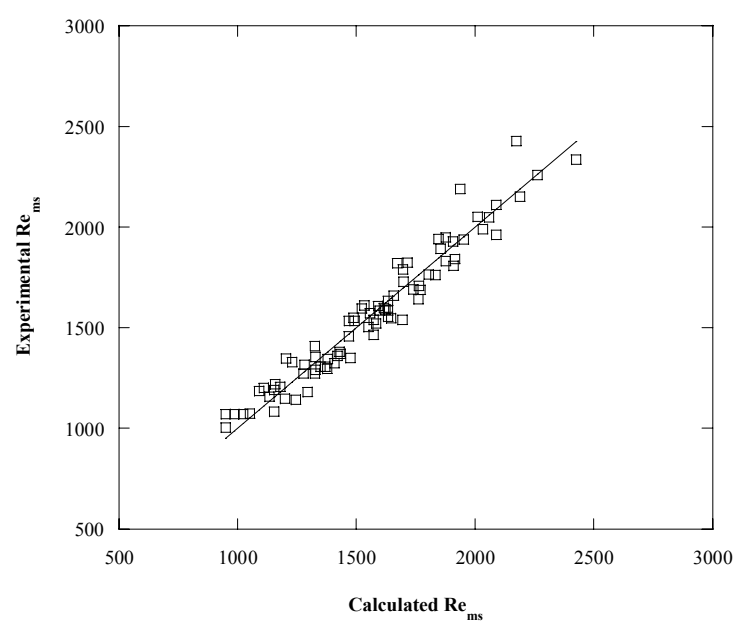

Figure 6. Correlation, straight line, experimental results, white squares.

The results of this correlation, as well as those that can be obtained from more general equations, like the equation of Mathur and Gishler presented in Mathur and Epstein (1974)

$$
U_{m s}=\left(\frac{d_{p}}{D}\right)\left(\frac{D_{i}}{D}\right)^{1 / 3} \sqrt{\frac{2 g H\left(\rho_{p}-\rho_{f}\right)}{\rho_{f}}}
$$

and from the equation of Nicolaev and Golubev (1964), more specific for conical spouted beds,

$$
\operatorname{Re}_{m s}=0.051 \mathrm{Ar} r^{0.59}\left(\frac{D_{i}}{D}\right)^{0.1}\left(\frac{H}{D}\right)^{0.25}
$$

were compared with the experimental results and the overall conclusions can be seen on Table 3 . It is evident that the correlation obtained in the present work is the best for conical spouted beds of cork stoppers.

Although the work of Markowski and Kaminski (1983) was a reference to define the operational conditions for the present work, the results for the minimum spouting velocity obtained with their correlation were far away from the experimental data obtained for cork stoppers. Consequently, the comparison among correlations has not taken into account the correlation of Markowski and Kaminski (1983). 
Table 3. Comparison among correlations.

\begin{tabular}{|l|c|c|}
\hline \multicolumn{1}{|c|}{ Correlations } & $\begin{array}{c}\text { Maximum } \\
\text { deviation } \\
(\%)\end{array}$ & $\begin{array}{c}\text { Average } \\
\text { deviation } \\
(\%)\end{array}$ \\
\hline This work, Eq. (3) & 11.5 & 5.2 \\
\hline Mathur and Gishler, Eq. (7) & 37.5 & 19.1 \\
\hline $\begin{array}{l}\text { Nicolaev and Golubev, Eq. } \\
(8)\end{array}$ & 31.1 & 14.1 \\
\hline
\end{tabular}

\section{CONCLUSIONS}

An experimental study on the definition of the minimum spouting conditions for a jet spouted bed of cork stoppers, has shown that, due to the peculiar physical characteristics of the cork stoppers, the curve of the evolution of the pressure drop versus gas superficial velocity is different from other granular materials. At the instant of minimum spouting there is only a slight change on the slope of that curve.

The experimental data for the minimum spouting velocity were correlated by a dimensionless equation and the maximum relative deviation is of $11.5 \%$ while an average deviation of $5.2 \%$ was found.

The number of tested experimental situations is limited and logically the range of applicability of such correlation is somehow restricted. However, the presently developed correlation leads to a better fitting of the minimum spouting velocity for a bed of cork stoppers, when compared with other correlations found in the literature.

\section{REFERENCES}

Costa, M. A. and Taranto, O. P., 2003, Scale-up and Spouting of Two-Dimensional Beds, The Canadian Journal of Chemical Engineering, Vol.81, pp. 264-267.

Geldart, D., 1986, Gas Fluidization Technology, John Wiley and Sons, New York.

Kmieg, A., 1983, The Minimum Spouting Velocity in Conical Beds, The Canadian Journal of Chemical Engineering, Vol.61, pp. 274-280.

Lefroy, G. A. and Davidson, J. F., 1969, The Mechanics of Spouted Beds, Trans. Instn. Chem. Engrs., Vol. 47, pp. T120-T128.

Littman, H. and Morgan III, M. H., 1983, A General Correlation for the Minimum Spouting Velocity, The Canadian Journal of Chemical Engineering, Vol.61, pp. 269-273.

Markowski, A. and Kaminski, W., 1983, Hydrodynamic Characteristics of Jet-Spouted Beds, The Canadian Journal of Chemical Engineering, Vol.61, pp. 377-381.

Martins, L. B., Pinho, C.M.C.T. and Santos, A. S., 1994, Characteristic Drying-Curves of Corks in a Slim Fixed Bed, $3^{\text {th }}$ North-Northeastern Congress of Mechanical Engineering, $21^{\text {st }}$ to $24^{\text {th }}$ November 1994, Belém, Pará, Brazil.
Mathur, N. B., and Epstein, N., 1974, Spouted Beds, Academic Press, Inc., New York.

Németh, J., Pallai, E. and Aradi, E., 1983, Scale-Up Examination of Spouted Bed Dryers, The Canadian Journal of Chemical Engineering, Vol.61, pp. 419-425.

Nikolaev, A. M. and Golubev, L. G., 1964, Basic hydrodynamic characteristics of the spouting bed, Izv. Vyssh. Ucheb. Zaved. Khim. Tekhnol., Vol. 7, p. 855.

Olazar, M., San José, M. J., Aguayo, A. T., Arandes, J. M. and Bilbao, J., 1993, Pressure drop in conical spouted beds, The Chemical Engineering Journal, Vol. 51, pp. 53-60.

Pinho, C.M.C.T. and Santos, A. S., 1997, Design and Development of a Continuous Cork Stoppers Dryer, $14^{\text {th }}$ Brazilian Congress of Mechanical Engineering, $8^{\text {th }}$ to $12^{\text {th }}$ December 1997 , Bauru, SP, Brazil.

Rangel, N., Santos, A. and Pinho, C., 2001, Pressure Drop in Packed Shallow Beds of Cylindrical Cork Stoppers, Trans. IChemE, Vol. 79, Part A, pp 547-552.

Rangel, N., Santos, A. and Pinho, C., 2001a, Friction and Inertia Effects for the Evaluation of Pressure Drop in Flow Through Packed Beds of Cork Stopper", Proceedings of COBEM 2001, Fluid Mechanics, Vol. 8, pp 124-129, 26 $6^{\text {th }}$ to $30^{\text {th }}$ November 2001, Uberlândia, MG, Brazil.

Viswanathan, K., 1986, Model for Continuous Drying of Solids in Fluidized/Spouted Beds, The Canadian Journal of Chemical Engineering, Vol.64, pp. 87-95.

Wen, C.Y. and Chen, L.H., 1982, Fluidized Bed Freeboard Phenomena: Entrainment and Elutriation, AIChE J., Vol. 28, No. 1, pp. 117-128.

Yang, W.C., 2003, Handbook of Fluidization and Fluid-Particle Systems, Marcel Dekker, Inc., New York.

Received: March 04, 2006

Revised: April 04, 2006

Accepted: May 04, 2006 